\title{
UTILIZACIÓN DE RESIDUOS AGRÍCOLAS Y AGROINDUSTRIALES EN EL CULTIVO Y PRODUCCIÓN DEL HONGO COMESTIBLE SETA (Pleurotus spp.)
}

\author{
Ana Belem PIÑA-GUZMÁN ${ }^{1,3}$, Diego Alejandro NIETO-MONTEROS² y Fabián ROBLES-MARTÍNEZ ${ }^{1,3 *}$ \\ ${ }^{1}$ Sección de Posgrado e Investigación, Unidad Profesional Interdisciplinaria de Biotecnología. Instituto Poli- \\ técnico Nacional. Av. Acueducto s/n, Barrio La Laguna, Col. Ticomán, D.F., C.P. 07340, México \\ ${ }^{2}$ Sección de Ingeniería Ambiental, Departamento de Química y Ciencias Exactas. Universidad Técnica Parti- \\ cular de Loja. San Cayetano alto s/n, C.P. 1101608, Loja, Ecuador \\ ${ }^{3}$ REDISA. Red de Ingeniería en Saneamiento Ambiental \\ *Autor para correspondencia: froblesm@ipn.mx
}

(Recibido octubre 2014; aceptado agosto 2015)

Palabras clave: biodegradación, eficiencia biológica (EB), materiales lignocelulósicos

\section{RESUMEN}

Algunos procesos productivos permiten el aprovechamiento de subproductos, desechos o residuos generados en otros procesos distintos. Lo anterior es interesante desde el punto de vista del desarrollo sustentable debido a que, por un lado, se tiende a aprovechar al máximo los recursos y, por otro, se disminuye la disposición de residuos. Un ejemplo de esto último es el cultivo de setas, en cuya producción pueden utilizarse como sustrato una gran variedad de residuos o subproductos agrícolas o agroindustriales. El cultivo de setas es un sistema biotecnológico eficiente, ya que se logran altos rendimientos y buena productividad con pocos controles ambientales. Las setas tienen tiempos de crecimiento cortos, crecen en un amplio intervalo de temperaturas y su habilidad para utilizar diversos materiales lignocelulósicos como sustrato, hace posible el empleo de residuos agrícolas, agroindustriales y forestales disponibles regionalmente. En México, el cultivo de setas es una actividad económica y ecológicamente importante, genera empleos y permite el reciclaje de más de 500000 toneladas anuales de residuos agrícolas, agroindustriales y forestales, aminorando así el impacto ambiental de la disposición final de dichos residuos. El objetivo del presente trabajo es presentar una revisión de las investigaciones realizadas principalmente en Iberoamérica, en relación a la utilización de diversos subproductos biológicos en el cultivo y producción de los hongos comestibles (Pleurotus spp.). Los materiales utilizables como sustratos para el cultivo de setas se clasifican en seis categorías: pajas, rastrojos, pulpas, bagazos, residuos forestales y otros. Se analiza la amplia variación en eficiencias biológicas (EB) (15\% al $235 \%$ ) obtenidas en los diferentes estudios y, de manera sintética, los criterios de elegibilidad de los materiales.

Key words: biodegradation, biological efficiency (BE), lignocellulosic substrates

\begin{abstract}
Some productive processes allow the utilization of by-products, waste or residues generated in other different processes. From the point of view of the sustainable development, this is interesting because, on the one hand, the resources are to be useful to the maximum, and for other one, the waste disposal decreases. A good example is the use of a great variety of agricultural crop residues and agroindustrial by-products as substrates in the production of edible mushrooms. Mushroom cultivation is an efficient
\end{abstract}


biotechnological process, since high performances and good productivity are achieved with low environmental controls. Mushrooms grow in a wide range of temperature, in short periods and has the ability of biodegrade different lignocellulosic substrates. This makes possible the valorization of agricultural, forest and agroindustrial residues regionally available. In Mexico, mushroom cultivation is an important economic and ecological activity, because it generates employments and enables the use and recycling of more than 500,000 tons of agricultural, agroindustrial and forest residues, per year. Moreover, it reduces the environmental impact of the final disposition of green residues. The goal of this work is to present a review of the research, mainly in Ibero-American countries, about the utilization of different green wastes (substrates) on the cultivation and production of edible mushrooms (Pleurotus spp.). Substrates were classified as straw, stubble, pulp, bagasse, forest residues, and others. The wide variation of biological efficiency (BE) (15\% - $235 \%)$ obtained in different studies, and the criteria needed to choose the correct substrate for the cultivation of mushrooms were also analyzed.

\section{APROVECHAMIENTO DE RESIDUOS AGRÍ- COLAS Y AGROINDUSTRIALES EN LA PRODUCCIÓN DE HONGOS COMESTIBLES}

Debido al rápido crecimiento de la agroindustria y a la extensión de terrenos cultivados en algunas regiones agrícolas, la cantidad de materiales orgánicos de desecho son una fuente de contaminación ambiental pues los grandes volúmenes generados rebasan la capacidad de biodegradación natural, de manera que estos desperdicios se pueden acumular llegando a convertirse en un riesgo para el equilibrio del ecosistema, ya sea porque son depositados en terrenos sin control alguno o bien quemados en el sitio generándose de esta manera contaminación ambiental. Por esta razón hay un interés creciente en la utilización de estos residuos en procesos productivos que permitan emplear estos materiales con los consecuentes beneficios económicos y ambientales.

El cultivo de hongos comestibles es una industria biotecnológica en continuo proceso de expansión y que va cobrando mayor importancia en el ámbito económico de muchos países. Entre los principales países productores de hongos comestibles se encuentra China, Estados Unidos, Holanda, Francia, España, Polonia, Italia, Canadá, Irlanda y Reino Unido (Sánchez 2010). En China hay una gran tradición por el consumo y el cultivo de hongos comestibles; sin embargo, en México, se tienen registrados más hongos silvestres comestibles (Andrade et al. 2013).

Los hongos Pleurotus ssp. son agentes biológicos de gran interés por su capacidad de convertir los subproductos orgánicos no comestibles en alimentos humanos de buena palatabilidad, con una eficiencia de conversión de proteína por unidad de área y por unidad de tiempo superior a las fuentes de proteína animal (Rodríguez y Zuluaga 1994). Este género se encuentra distribuido en Europa, Asia, Australia y América y, Pleurotus ostreatus es el segundo hongo comestible más cultivado por todo el mundo después de Agaricus bisporus (Sánchez 2010). En todo América (incluidos Canadá y Estados Unidos), México es el principal país productor de Pleurotus spp., solamente seguido por Brasil (Andrade et al. 2013). En Iberoamérica se han realizado esfuerzos científicos y tecnológicos por probar nuevos materiales como sustrato para el cultivo del hongo. El objetivo del presente documento es dar a conocer de forma sintetizada diferentes trabajos de Iberoamérica, en los que se ha investigado una gran variedad de materiales como posibles soportes en el cultivo y producción de los hongos comestibles setas (Pleurotus spp.).

\section{CARACTERÍSTICAS GENERALES DE LAS SETAS (Pleurotus spp.)}

El género Pleurotus comprende especies comestibles de excelente gusto y sabor que generalmente son de color blanco, amarillento o rosado, a veces grisáceo o de color oscuro. A los hongos de este género se les denomina seta, aunque éstos también son conocidos popularmente como hongos ostra, orejas blancas, orejas de palo, orejas de patancán, orejas de cazahuate y orejas de izote (Gaitán-Hernández et al. 2006). Hasta ahora se han registrado aproximadamente 70 especies del género Pleurotus y se siguen descubriendo nuevas especies.

Los hongos del género Pleurotus han visto incrementada su popularidad a nivel mundial en los últimos años, debido a su capacidad para crecer en un amplio intervalo de temperaturas y su habilidad para utilizar como sustrato diversos materiales ricos en lignina y celulosa (Melo de Carvalho et al. 2010). 
Requieren menor tiempo de crecimiento en comparación con otros hongos comestibles, exigen pocos controles ambientales y sus cuerpos fructíferos no son a menudo atacados por plagas y enfermedades (Jwanny et al. 1995). Otras ventajas, además de su fácil cultivo, son su alto rendimiento de productividad y su elevado valor nutricional por su alto contenido en proteínas de buena calidad, vitaminas, ácidos grasos insaturados y elementos esenciales como calcio, hierro, magnesio, fósforo, potasio, sodio, zinc, cobre, manganeso y selenio, con bajo contenido calórico y libre de colesterol (Kumari y Achal 2008). Además, los extractos o polvos provenientes de los cuerpos fructíferos de Pleurotus han sido reconocidos por poseer propiedades medicinales, tales como: antioxidante, hepatoprotectora, antidiabética, anticancerígena, entre otras (Asaduzzaman y Mousumi 2012).

\section{EL CULTIVO DEL GÉNERO Pleurotus}

Los hongos del genero Pleurotus spp. son saprófitos, crecen de manera natural sobre troncos, ramas o árboles muertos y algunas veces se encuentra en el suelo sobre raíces podridas (Díaz 2009). Gracias a estas propiedades, Pleurotus spp. puede crecer también de manera controlada en diferentes materiales obtenidos como subproductos o desechos de las actividades agrícolas y agroindustriales, lo cual trae un beneficio ambiental debido a que el manejo y el tratamiento adecuado de desechos orgánicos en el mundo cada día resulta más necesario.

Guzmán et al. (2008) señalan que los materiales elegibles para ser utilizados en la preparación de sustratos para el cultivo de Pleurotus spp., deben poseer el mayor número posible de propiedades favorables tales como: buena disponibilidad en cantidad y continuidad; características físico-químicas conocidas; regularidad en su composición físico-química; precio de adquisición ventajoso; localización fácil y cercana al sitio de uso y facilidad de trasporte y manejo. De acuerdo al mismo autor, los sustratos para el cultivo de los hongos se pueden clasificar en seis categorías, a saber: 1) Pajas (de ajonjolí, arroz, cártamo, cebada, sorgo, trigo, avena y zacate en general); 2) Rastrojos (de maíz, mijo, garbanzo, frijol, etc.; 3) Pulpas (de café, de limón, de cardamomo); 4) Bagazos (de caña de azúcar, citronela, maguey tequilero, henequén, uva, etc.); 5) Residuos forestales (como aserrín, viruta, troncos y ramas); y 6) Otros (como papel, olote, tamo de maíz, hojas de piña, fibra de coco, lirio acuático, hojas y tallos de plátano, desechos de la industria textil, etc.).
La productividad del cultivo de los hongos se valora mediante el parámetro de eficiencia biológica (EB) expresado en porcentaje. La EB se expresa en $\mathrm{kg}$ de biomasa de hongos por $\mathrm{kg}$ de sustrato, y resulta de dividir el peso fresco de los hongos cosechados entre el peso seco del sustrato empleado. Con la EB se puede evaluar la calidad de un residuo orgánico como sustrato para el cultivo de hongos, de tal forma que cuanto mayor sea el valor de este parámetro, mayor será la idoneidad del sustrato para el cultivo de la cepa de hongo en particular (Ragunathana y Swaminathan 2003). Ríos et al. (2010) indican que una EB se percibe como aceptable a partir del $50 \%$, ya que este es el valor mínimo de referencia para el cultivo comercial de $P$. ostreatus considerado como económicamente rentable. Aunque una EB adecuada debe ser cercana o mayor al $100 \%$, los productores de hongos no sólo están interesados en la obtención de altos rendimientos, sino también en ofrecer un buen producto de alta calidad, donde la morfología y el color de los cuerpos fructíferos, y el tamaño del píleo y estípite son también aspectos importantes a tener en cuenta cuando los agricultores seleccionan 1 "semilla".

\section{DEGRADACIÓN DE COMPUESTOS LIGNO- CELULÓSICOS}

La lignocelulosa es el componente principal de la biomasa vegetal y es el más abundante de los recursos renovables. Se compone de tres tipos de polímeros: celulosa (principal componente), hemicelulosa y lignina, que están fuertemente entrelazados y unidos químicamente por fuerzas no covalentes y reticulaciones covalentes (Pérez et al. 2002), formando un sello físico en la pared celular de las plantas que es una barrera impenetrable. La celulosa y la hemicelulosa son macromoléculas construidas a partir de diferentes azúcares. La lignina es un heteropolímero amorfo, insoluble en agua y ópticamente inactivo, se forma a partir de subunidades de fenilpropano interconectadas mediante enlaces no hidrolizables carbono-carbono y carbono-oxígeno-carbono (Sánchez 2009); está presente en la pared celular para dar soporte estructural, impermeabilidad y resistencia contra el ataque microbiano y el estrés oxidativo. El principal enlace de la estructura de la lignina es el éter arilglicerol- $\beta$-aril, enlace que comprende la mitad de los enlaces de la estructura (Young y Akhtar 1998). La composición y las proporciones de estos tres compuestos varían entre cada planta (Sánchez 2009). Muchos microorganismos son capaces de degradar la celulosa y la hemicelulosa para utilizarlos como 
fuente de carbono y energía. Sin embargo, un grupo muy reducido de hongos filamentosos han evolucionado en su capacidad para descomponer la lignina, que es el componente más recalcitrante de la pared celular de las plantas. Estos hongos son conocidos como los hongos de pudrición blanca (PB), entre los cuales se encuentran los del género Pleurotus, y que poseen la capacidad única de degradar de manera eficiente la lignina a $\mathrm{CO}_{2}$ y agua (Hurst et al. 2007).

Durante el crecimiento y el desarrollo del micelio, los cambios bioquímicos tienen lugar como consecuencia de la producción de enzimas extracelulares. Estas enzimas degradan los componentes insolubles y gran cantidad de material lignocelulósico en compuestos solubles y de bajo peso molecular, que son tomados posteriormente por enzimas intracelulares del hongo para su nutrición. Por lo tanto, las enzimas tienen un papel significativo en el crecimiento y desarrollo de hongos (Kurt y Buyukalaca 2010). Los hongos tienen dos tipos de sistemas enzimáticos extracelulares: el sistema de hidrólisis, que produce hidrolasas que son responsables de la degradación de polisacáridos, y un sistema ligninolítico oxidativo único, que degrada lignina y abre los anillos de fenilo (Sánchez 2009).
El sistema enzimático ligninolítico es poco específico, ya que las peroxidasas y oxidasas actúan al azar sobre la molécula de lignina, generando radicales libres que son inestables y tienden a polimerizarse. Las principales enzimas que actúan directa o indirectamente sobre la lignina son: lignina peroxidasas (LiP), manganeso peroxidasas (MnP) y lacasas (Young y Akhtar 1998). Los hongos de la pudrición blanca, secretan una o más de las tres enzimas extracelulares oxidativas esenciales en la mineralización de lignina: la LiP que, por síntesis endógena de $\mathrm{H}_{2} \mathrm{O}_{2}$, oxida el veratril alcohol y compuestos aromáticos no fenólicos, en una reacción que genera radicales arilo y alquilo que se anabolizan intracelularmente; la MnP, que oxida componentes fenólicos de la lignina, mediante la reacción de oxidación del $\mathrm{Mn}^{2+}$ a $\mathrm{Mn}^{3+}$, la cual es dependiente del $\mathrm{H}_{2} \mathrm{O}_{2}$; y la lacasa, una fenol oxidasa con cobre, que oxida anillos de la lignina (Pérez et al. 2002). Todas estas enzimas participan en la degradación bajo condiciones de fermentación en estado sólido, y se ha detectado que ayudan tanto a la infestación del micelio sobre el sustrato como a la degradación del mismo (Kudryavtseva et al. 2008).

CUADRO I. PAÍSES DE IBEROAMÉRICA DONDE SE HAN REPORTADO TRABAJOS DE INVESTIGACIÓN SOBRE EL USO DE RESIDUOS AGRÍCOLAS, AGROINDUSTRIALES Y FORESTALES EN LA PRODUCCIÓN DE Pleurotus spp.

\begin{tabular}{|c|c|c|c|}
\hline País & Referencia & País & Referencia \\
\hline Colombia & $\begin{array}{c}\text { Rodríguez y Zuluaga 1994, Bonilla y } \\
\text { López 2001, Sánchez-Velez 2013, López- } \\
\text { Rodríguez et al. } 2008 \text {, Rivera-Omen et } \\
\text { al. } 2013\end{array}$ & Brasil & $\begin{array}{l}\text { Moda et al. } 2005 \\
\text { Loss et al. } 2009\end{array}$ \\
\hline Cuba & Bermúdez et al. 2001 y 2007 & Ecuador & Toledo 2008 \\
\hline Argentina & $\begin{array}{l}\text { Curvetto et al. 2002, Fracchia et al. 2009, } \\
\text { Lechner y Albertó } 2011\end{array}$ & México-Francia & Velázquez-Cedeño et al. 2002 \\
\hline
\end{tabular}

Soto-Velazco et al. 1989, Lara et al. 2002, Bernabé-González et al. 2004, Salmones et al. 2004 y 2005, Pérez y Mata México 2005, García et al. 2006, Guzmán et al. 2008, Sánchez et al. 2008, Gaitán-Hernández et al. 2009, Sánchez-Vélez 2013,

Romero et al. 2010, Flores 2012.
América Central (Guatemala,

República Dominicana, El Salvador, Cruz et al. 2010 Honduras, Costa Rica y Nicaragua)

\begin{tabular}{lll} 
España & Andrino et al. 2011 & Chile \\
\hline
\end{tabular}




\section{RESIDUOS AGROINDUSTRIALES EMPLEA- DOS EN EL CULTIVO DE Pleurotus spp.}

Puesto que Pleurotus spp. puede descomponer la lignocelulosa de manera eficiente sin un pretratamiento biológico o químico, para su cultivo se han utilizado una gran variedad de desechos lignocelulósicos, de los que la mayoría se han empleado como alimentos para animales pero, debido a su baja digestibilidad, bajo contenido de proteína y alto contenido de lignina no son recomendables para tal fin (Cohen et al. 2002). Tradicionalmente el género Pleurotus ha sido cultivado sobre paja de cereales como trigo, arroz, avena y cebada. En Asia, donde el arroz es cultivado en grandes extensiones, la paja de arroz es ampliamente aceptada como sustrato principal para cultivar hongo ostra y así se aprovecha este residuo agrícola. Por otro lado, varios autores coinciden en que para el cultivo de Pleurotus spp. la paja de trigo es el mejor sustrato por su disponibilidad y economía (Kumari y Achal 2008), encontrándose que en la producción comercial de diferentes especies y cepas de Pleurotus, los valores de EB varían entre $30.5 \%$ (Salmones et al. 2005) y $161.7 \%$ (Lechner y Albertó 2011). Sin embargo, en vista de la creciente demanda, costo y otros usos alternativos de la paja de arroz o trigo, se ha considerado la posibilidad de uso de otros residuos lignocelulósicos sin valor comercial, que se tengan disponibles en cada localidad y que puedan ser aprovechados como nuevos sustratos para la producción comercial de hongos ostra con alto contenido de proteínas, generando así productos con valor económico y contribuyendo de esta manera al cuidado del ambiente. El cuadro I muestra una lista de diferentes países de Iberoamérica en donde se han realizado trabajos de investigación en los que se han probado diferentes residuos agrícolas, agroindustriales y forestales como sustratos para la producción de Pleurotus spp.

Teniendo en cuenta que en México se está incrementando el interés por la producción comercial de hongos comestibles y se busca el abaratamiento de los costos de producción mediante el aprovechamiento de residuos lignocelulósicos disponibles en cada región, es importante la búsqueda de sustratos alternativos de bajo costo y fácil acceso, sin dejar de lado que para el cultivo exitoso es fundamental la plena identificación del material que asegure que el sustrato resultará adecuado, tanto biológica como económicamente (Tisdale et al. 2006). Por tal motivo, hay un creciente interés en la búsqueda de nuevos sustratos o mezclas que ofrezcan una alta disponibilidad de nutrientes para el cultivo de Pleurotus y que aseguren mayores rendimientos. En el cuadro II se muestra una lista de materiales probados como sustratos y la Eficiencia Biológica (EB) obtenida para unas 10 de las más de 70 especies conocidas de Pleurotus spp. Entre los materiales probados se encuentran diferentes residuos de diferentes actividades productivas como la agrícola, forestal, agroindustrial y especies vegetales originarias del lugar donde los estudios fueron realizados. Tal es el caso de la quinua, un pseudocereal originario de la región andina de Perú, Ecuador, Bolivia y Colombia, cuyo rastrojo solo y combinado con olote de maíz ha sido probado (Toledo 2008), o bien el maguey tequilero originario de México, cuyo bagazo seco y fermentado han sido probados como sustratos (Lara et al. 2002, Bernabé-González et al. 2004), obteniéndose EB aceptables (>50\%). En lo que respecta al bagazo de caña de azúcar, un residuo agroindustrial lignocelulósico producido en grandes volúmenes en países como Brasil y México, ha sido ya probado en el cultivo de Pleurotus sajor-caju por Moda et al. (2005), obteniendo EB de 13.9 - $19.2 \%$.

El cuadro III muestra algunas combinaciones en las que diversos residuos fueron empleados como cosustratos, donde se mezclaron con sustratos típicos (olote de maíz, paja de trigo, paja de arroz, rastrojo de maíz) e incluso fueron suplementados con efluentes residuales de origen agroindustrial. En la mayoría de los casos, dichas combinaciones ofrecen mejores rendimientos que los residuos solos (co-sustratos). En un estudio realizado por Flores (2012), la combinación de bagazo de yuca + paja de trigo tuvo un mayor rendimiento en comparación con el bagazo de yuca solo. De manera similar, Rajak et al. (2011) reportaron que con la combinación de paja de arroz (sustrato) y hierbas silvestres de la India (co-sustrato) se obtuvo el mayor rendimiento, en comparación con las hierbas silvestres solas. Esto podría ser explicado por el hecho de que en la mezcla, los sustratos típicos aportan los nutrientes necesarios para la incubación y desarrollo de primordios y formación de esporóforos durante la colonización, mientras que los co-sustratos, cuya descomposición es más lenta que la de los sustratos, proveen los requerimientos nutricionales necesarios para los estadíos posteriores de crecimiento. De manera contraria, algunas combinaciones de sustratos resultan desfavorables para la producción de Pleurotus. Tal es el caso del estudio realizado por Bermúdez et al. (2007), en el que la EB obtenida con Pleurotus sajor-caju sobre la combinación de pulpa de café + viruta de cedro (136.9 \%) no logra superar la extraordinaria EB alcanzada por la pulpa de café sola (225.2\%). En este estudio, la pobreza de 
CUADRO II. EFICIENCIA BIOLÓGICA DE DISTINTAS ESPECIES DEL GÉNERO Pleurotus SOBRE DIVERSOS RESIDUOS AGROINDUSTRIALES.

\begin{tabular}{|c|c|c|c|c|}
\hline & $\begin{array}{l}\text { Residuos utilizados } \\
\text { como sustrato }\end{array}$ & Especies de Pleurotus & EB (\%) & Referencia \\
\hline \multirow{6}{*}{$\cdot \frac{\pi}{\pi}$} & De trigo & $\begin{array}{l}\text { P. ostreatus, } P . \text { florida, } \\
\text { P. sajor-caju, P. albidus, } \\
\text { P. pulmonarius, P. eryngii, } \\
\text { P. djamor }\end{array}$ & $30.5-161.7$ & $\begin{array}{l}\text { Philippoussis et al. 2001, Salmones et al. } \\
\text { 2005, Ahmed et al. 2009, Varnero et al. } \\
\text { 2010, Fanadzo et al. 2010, Romero et al. } \\
\text { 2010, Kurt y Buyukalaca 2010, Flores } \\
\text { 2012, Lechner y Albertó } 2011\end{array}$ \\
\hline & De arroz & $\begin{array}{l}\text { P. ostreatus, } \boldsymbol{P} \text {. florida, } \\
\text { P. sajor-caju, }\end{array}$ & $50.6-75.1$ & $\begin{array}{l}\text { Basak et al. 1996, Obodai et al. 2003, } \\
\text { Ahmed } \text { et al. 2009, Kurt y Buyukalaca } \\
2010\end{array}$ \\
\hline & De cebada & $\begin{array}{l}\text { P. ostreatus, P. pulmonarius, } \\
\text { P. djamor }\end{array}$ & $66.3-124.88$ & $\begin{array}{l}\text { Salmones et al. 2004, Pérez y Mata 2005, } \\
\text { Romero et al. } 2010\end{array}$ \\
\hline & De soja & P. florida, P.eous, & $76.8-87.56$ & Ahmed et al. 2009, Ingale y Ramteke 2010 \\
\hline & De frijol & P. ostreatus & 76.95 & Romero et al. 2010 \\
\hline & De sésamo & P. ostreatus, $P$. sajor-caju & $66.2-91.3$ & Kurt y Buyukalaca 2010 \\
\hline \multirow{6}{*}{ 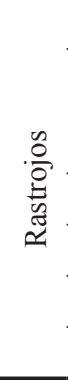 } & De algodón & P. ostreatus, $P$. pulmonarius & $70.61-116.7$ & Philippoussis et al. 2001 \\
\hline & De maíz & $\begin{array}{l}\text { P. ostreatus, } P \text {. sajor-caju, } \\
\text { P. pulmonarius, } \text { P. floridae }\end{array}$ & $19.9-154.1$ & $\begin{array}{l}\text { Obodai et al. 2003, Bernabé-González et } \\
\text { al. 2004, Loss et al. 2009, Romero et al. } \\
\text { 2010, Fanadzo et al. 2010. }\end{array}$ \\
\hline & De tomate & P. ostreatus & 118.5 & Sánchez et al. 2008, \\
\hline & De sorgo & P. sajor-caju & 36.8 & Ragunathan y Swaminathan 2003, \\
\hline & De jamaica (S y F) & P. pulmonarius & $44.1-78.4$ & Bernabé-González et al. 2004 \\
\hline & De quinua & P. florida & 32 & Toledo 2008 \\
\hline \multirow{3}{*}{$\frac{\mathscr{2}}{\Xi}$} & De café & $\begin{array}{l}\text { P. ostreatus, } P \text {. sajor-caju, } \\
\text { P. pulmonarius, } P \text {. djamor }\end{array}$ & $67.1-225.2$ & $\begin{array}{l}\text { Bermúdez et al. 2001, 2007, Velázquez- } \\
\text { Cedeño et al. 2002, Salmones et al. 2005, } \\
\text { García et al. 2006, Huerta et al. } 2009\end{array}$ \\
\hline & De limón & P. ostreatus & 5 & Rashad et al. 2009 \\
\hline & De cardamomo & P. ostreatus & 113.64 & Morales 1987 \\
\hline \multirow{5}{*}{ 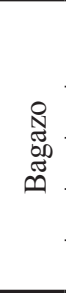 } & $\begin{array}{l}\text { De maguey tequilero } \\
(\mathrm{S} \text { y F })\end{array}$ & $\begin{array}{l}\text { P. ostreatus, } P \text {. sajor-caju, } \\
\text { P. pulmonarius }\end{array}$ & $69.1-78.3$ & $\begin{array}{l}\text { Lara et al. 2002, Bernabé-González et al. } \\
\mathbf{2 0 0 4}\end{array}$ \\
\hline & De caña de azúcar & P. sajor-caju & $13.9-19.2$ & Moda et al. 2005 \\
\hline & De cervecera & P. eryngii & $41.02-87.35$ & Andrino et al. 2011 \\
\hline & De yuca & P. ostreatus & $54.7-69.4$ & Flores 2012 \\
\hline & De remolacha azucarera & P. ostreatus & 111.8 & Jafarpour et al. 2010 \\
\hline \multirow{4}{*}{ 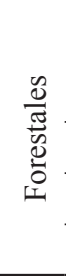 } & $\begin{array}{l}\text { Aserrín } \\
\text { Viruta de madera }\end{array}$ & $\begin{array}{l}\text { P. columbinus, P. ostreatus, } \\
\text { P. albidus }\end{array}$ & $47.2-135.6$ & $\begin{array}{l}\text { Obodai et al. 2003, Narh et al. 2011, Lech- } \\
\text { ner y Albertó 2011, Jafarpour } \text { et al. 2010, } \\
\text { Mandeel et al. 2005 }\end{array}$ \\
\hline & Viruta de cedro & P. ostreatus, P. sajor-caju & $68.5-89.4$ & Bermúdez et al. 2007 \\
\hline & Viruta de pino & P. ostreatus, $p$. pulmonarius & $27.9-53.5$ & Pérez y Mata 2005 \\
\hline & Astillas de eucalipto & P. ostreatus & 4.2 & Varnero et al. 2010 \\
\hline \multirow{3}{*}{$\stackrel{n}{0}$} & $\begin{array}{l}\text { Residuos de maíz (hojas } \\
\text { y olotes) }\end{array}$ & $\begin{array}{l}\text { P. ostreatus, } \boldsymbol{P} \text {. florida, } \\
\text { citrinopileatus }\end{array}$ & $16.5-78.8$ & $\begin{array}{l}\text { Obodai et al. 2003, Toledo 2008, Naraian } \\
\text { et al. 2009, Liang et al. 2009, Mamiro y } \\
\text { Mamiro } 2011\end{array}$ \\
\hline & Hierbas de la india & P. sajor-caju & & Rajak et al. 2011 \\
\hline & $\begin{array}{l}\text { Malezas y especies } \\
\text { arbustivas del bosque }\end{array}$ & P. ostreatus & $22.9-97.9$ & $\begin{array}{l}\text { Tisdale et al. 2006, Das y Mukherjee 2007, } \\
\text { Fracchia et al. } 2009\end{array}$ \\
\hline
\end{tabular}




\section{CUADRO II. (cont.) EFICIENCIA BIOLÓGICA DE DISTINTAS ESPECIES DEL GÉNERO Pleurotus SOBRE DIVERSOS} RESIDUOS AGROINDUSTRIALES.

\begin{tabular}{|c|c|c|c|}
\hline $\begin{array}{l}\text { Pastos forrajeros } \\
\text { (Panicum virgatum L., } \\
\text { P. repens, Pennisetum } \\
\text { purpureum, Hyparrhe- } \\
\text { nia filipéndula }\end{array}$ & $\begin{array}{l}\text { P. ostreatus, } P \text {. cornucopiae; } \\
\text { P. citrinopileatus }\end{array}$ & $5.3-57.3$ & $\begin{array}{l}\text { Royse et al. 2004, Liang et al. 2009, Fanad- } \\
\text { zo et al. 2010, Mamiro y Mamiro } 2011\end{array}$ \\
\hline $\begin{array}{l}\text { Capuchones, cáscaras } \\
\text { y cascarillas (de cacao, } \\
\text { coco, cacahuate, semilla } \\
\text { de girasol, arroz, uchuva, } \\
\text { algodón, arveja) }\end{array}$ & $\begin{array}{l}\text { P. ostreatus, } P \text {. eryngii, } \\
\text { P. pulmonarius, } P \text {. citrinopi- } \\
\text { leatus }\end{array}$ & $7.8-120.7$ & $\begin{array}{l}\text { Bermúdez et al. 2001, Philippoussis et al. } \\
\text { 2001, Obodai et al. 2003, López-Rodríguez } \\
\text { et al. 2008, Rodríguez Estrada et al. } 2009 \text {, } \\
\text { Liang et al. 2009, Jafarpour et al. } 2010\end{array}$ \\
\hline Papel y cartón & P. ostreatus, P. colombinus & $100.8-134.5$ & Mandeel et al. 2005 \\
\hline $\begin{array}{l}\text { Fibras (de palma, yute, } \\
\text { coco, sisal) }\end{array}$ & $\begin{array}{l}\text { P. ostreatus, } P \text {. sajor-caju, } \\
\text { P. flabellatus, } P \text {. columbinus }\end{array}$ & $27.3-97.2$ & $\begin{array}{l}\text { Basak et al. 1996, Ragunathan y Swami- } \\
\text { nathan 2003, Mandeel et al. 2005, Mshan- } \\
\text { dete y Cuff 2008, Jafarpour et al. } 2010\end{array}$ \\
\hline $\begin{array}{l}\text { Hojas y tallos (plátano, } \\
\text { algodón, maíz, brócoli y } \\
\text { coliflor) }\end{array}$ & $\begin{array}{l}\text { P. ostreatus, } P \text {. sajor-caju, } \\
\text { P. citrinopileatus, P. florida }\end{array}$ & $37.2-123.3$ & $\begin{array}{l}\text { Basak et al. 1996, Ragunathan y Swami- } \\
\text { nathan 2003, Obodai et al. 2003, Liang } \\
\text { et al. 2009, Romero et al. 2010, Heredia } \\
\text { 2010, }\end{array}$ \\
\hline Residuos vitivinícolas & P. ostreatus, P. sajor-caju & $70.4-73.6$ & Kurt y Buyukalaca 2010 \\
\hline
\end{tabular}

Nota: En negritas se presentan los estudios en los que se obtuvieron las mayores EB. S: sustrato seco; F: sustrato fermentado

CUADRO III. EFICIENCIA BIOLÓGICA DE DISTINTAS ESPECIES DEL GÉNERO Pleurotus SOBRE DIVERSAS MEZCLAS DE RESIDUOS AGROINDUSTRIALES

\begin{tabular}{|c|c|c|c|}
\hline Mezclas de residuos utilizadas como sustrato & Especies de Pleurotus & EB $(\%)$ & Referencia \\
\hline $\begin{array}{l}\text { Bagazo de maguey tequilero (Agave tequilana) fermentado }+ \\
\text { paja de trigo }\end{array}$ & P. ostreatus & 96.4 & $\begin{array}{l}\text { Soto-Velazco et al. } \\
1989\end{array}$ \\
\hline Pulpa de café + viruta de cedro & P. sajor-caju & 136.9 & Bermúdez et al., 2007 \\
\hline Pseudotallo + hojas frescas de plátano (Musa paradisiaca) & P. ostreatus & 99.8 & \multirow{3}{*}{$\begin{array}{l}\text { Cayetano-Catarino y } \\
\text { Bernabé-González } 2008\end{array}$} \\
\hline $\begin{array}{l}\text { Tallo de jamaica (Hibiscus sabdariffa) + Paja de arroz } \\
\text { (Oryza sativa) }(2: 1)\end{array}$ & \multirow{2}{*}{ P.pulmonarius } & 74.4 & \\
\hline $\begin{array}{l}\text { Pseudotallo + hojas de plátano (Musa paradisiaca) } \\
\text { fermentado } 14 \text { días }\end{array}$ & & 96.4 & \\
\hline Rastrojo de quinua + olote de maíz (70:30) & P. florida & 96.67 & Toledo 2008 \\
\hline Pulpa de limón + cascarilla de arroz (1:1) & P. ostreatus & 26.98 & Rashad et al. 2009 \\
\hline Fruta papaya + cascarilla de arroz $(2: 1)$ & & 13.5 & \\
\hline Jatropha macrocarpa + paja de trigo & P. ostreatus & 76.4 & Fracchia et al. 2009 \\
\hline Paja de soja + Paja de arroz & \multirow{2}{*}{ P. florida } & 85.20 & Ahmed et al. 2009 \\
\hline Paja de soja + Paja de trigo & & 78.26 & \\
\hline Olote de maíz + pulpa de café $(3: 1)$ & P. ostreatus & 43.48 & Cruz et al. 2010 \\
\hline $\begin{array}{l}\text { Olote de maíz + Paja de trigo, suplementado con agua resi- } \\
\text { dual de industria de lácteos }\end{array}$ & P. florida & 108.68 & Naraian et al. 2011 \\
\hline Bagazo de yuca + paja de trigo & P. ostreatus & $54.7-69.4$ & Flores 2012 \\
\hline Fibra de coco + residuos de poda (diferentes proporciones) & P. ostreatus & $2.95-11.9$ & Sánchez-Velez 2013 \\
\hline
\end{tabular}


nitrógeno en la viruta de cedro puede ser la causante de los valores relativamente bajos de producción y fructificación obtenid os sobre dicha mezcla. Resulta también interesante mencionar que Delfín-Alcalá y Durán de Bazúa (2003) probaron paja de trigo y pasto mezclados con penachos de piña y posos de café (café lavado), estos tres últimos materiales tomados como parte de los residuos lignocelulósicos que pueden encontrarse en los residuos sólidos urbanos (RSU). Sin embargo, cabe remarcar la inconveniencia del uso de materiales recuperados de los RSU como sustratos para el cultivo de Pleurotus, dada la heterogeneidad de los materiales, los costos de separación, la contaminación de la materia orgánica por las demás fracciones contenidas en los RSU y la posible dificultad de acopiar cantidades suficientemente grandes para llevar a cabo un cultivo comercial sostenido.

Por último, es de gran importancia señalar que al final del cultivo de las setas, el sustrato agotado (SMS-spent mushroom substrate, por sus siglas en Inglés), si se enriquece con otros desechos agroindustriales puede ser empleado para el re-cultivo de más setas (Pleurotus spp.) o de otros hongos comestibles, o bien para la recuperación de suelos contaminados y áreas deforestadas, reduciendo de esta manera el impacto ambiental que puede provocar el SMS (Sánchez 2010).

\section{CONCLUSIONES}

Para el cultivo y producción de hongos del género Pleurotus, es posible el aprovechamiento de materiales residuales muy variados, lo cual permitiría destinar una parte de los sustratos convencionales como la paja de trigo y arroz, a otros usos alternativos como la alimentación animal y/o generación de energía. En Iberoamérica, en los últimos años, se han visto incrementados los estudios para la utilización de distintos residuos agrícolas y agroindustriales, ya sea solos o en combinación, con el fin de encontrar el material más adecuado que genere una alta productividad así como una mayor calidad en los hongos obtenidos a partir de él, encontrándose resultados alentadores que permitirán obtener ventajas en los ámbitos sociales, económicos y ambientales.

Los resultados de eficiencia biológica (EB) varían mucho de sustrato a sustrato, sin embargo los sustratos recomendables son aquellos que tienen un valor de EB cercano o mayor a 100, lo cual puede lograrse probando algunas combinaciones de materiales residuales o bien realizando algún tipo de pretratamiento del material como la fermentación, el composteo o simplemente la pasteurización. De esta manera, se pueden aprovechar residuos producidos regionalmente, para producir alimentos de alto contenido proteico y el sustrato agotado puede aún ser empleado tanto para el re-cultivo de hongos comestibles como para la recuperación de suelos contaminados, ofreciendo así alternativas tanto para la seguridad alimentaria como para la disminución del impacto ambiental ocasionado por los residuos agrícolas y agroindustriales.

\section{REFERENCIAS}

Ahmed S. A., Kadam J. A., Mane V. P., Patil S. S. y Baig M. M. V. (2009). Biological Efficiency and Nutritional Contents of Pleurotus florida (Mont.) Singer Cultivated On Different Agro-wastes. Nat. Sci. 7, 44-48.

Andrade R. H., Mata G. y Sánchez J. E. (2013). La producción iberoamericana de hongos comestibles en el contexto internacional. En: Hongos Comestibles y Medicinales en Iberoamérica: investigación y desarrollo en un entorno multicultural. (Sánchez Velázquez J.E. y Mata G., Eds.) ECOSUR-INECOL, México, pp. 393.

Andrino A., Morte A. y Honrubia M. (2011). Caracterización y cultivo de tres cepas de Pleurotus eryngii (Fries) Quélet sobre sustratos basados en residuos agroalimentarios. An. Biol. 33, 53-66.

Asaduzzaman K. y Mousumi T. (2012). Nutritional and Medical importance of Pleurotus mushrooms: an overview. Food Rev. Int. 28, 313-329. http://dx.doi.or g/10.1080/87559129.2011.637267

Basak M. K., Chanda S., Bhaduri S. K., Mondal S. B. y Nandi R. (1996). Recycling of jute waste for edible mushroom production. Ind. Crops Prod. 5, 173-176. DOI: 10.1016/0926-6690(00)00002-9

Bermúdez R. C., García N., Gross P. y Serrano M. (2001). Cultivation of Pleurotus on agricultural substrates in Cuba. Micol. Apl. Int. 13, 25-29.

Bermúdez R. C., García N. y Mourlot A. (2007). Fermentación sólida para la producción de Pleurotus spp. sobre mezclas de pulpa de café y viruta de cedro. Rev. Tecnol. Quim. 27, 55-62.

Bernabé-González T., Cayetano-Catarino M., Adán-Díaz A. y Torres-Pastrana M. A. (2004). Cultivo de Pleurotus pulmonarius sobre diversos subproductos agrícolas de Guerrero, México. Rev. Mex. Micol. 18, 77-80.

Bonilla E. Y. y López F. Y. (2001). Evaluación de la eficiencia biológica de dos cepas del hongo comestible Pleurotus ostreatus (Jaques ex Fríes) Quélet, en residuos postcosecha de caña de azúcar (Saccharum oficcinarum) y guayaba (Psidium guajava) en La Hoya del Río Suárez. Tesis de pregrado. Facultad de 
Ciencias. Escuela de Ciencias Biológicas. Universidad Pedagógica y Tecnológica de Colombia. Tuja, Colombia, 139 pp.

Cayetano-Catarino M. y Bernabé-González T. (2008). Cultivo de Pleurotus sobre residuos de las cosechas de jamaica (Hibiscus sabdariffa) y plátano (Musa paradisiaca). Rev. Mex. Micol. 26, 57-60.

Cohen R., Persky L. y Hadar Y. (2002). Biotechnological applications and potential of wood-degrading mushrooms of the genus Pleurotus. Appl. Microbiol. Biotechnol. 58, 582-594. DOI: 10.1007/s00253-002-0930-y

Cruz D., López de León E., Pascual L. F. y Battaglia M. (2010). Guía técnica de producción de hongos comestibles de la especie Pleurotus ostreatus. J. Agr. Environ. Int. Dev. 104, 139-154. DOI: http://dx.doi. org/10.12895/jaeid.20103/4.16

Curvetto N. R., Figlas D., Devalis R. y Delmastro S. (2002). Growth and productivity of different Pleurotus ostreatus strains on sunflower seed hulls supplemented with $\mathrm{N}-\mathrm{NH}^{+}{ }_{4}$ and/or $\mathrm{Mn}(\mathrm{II})$. Bioresource Technol. 84, 171-176. http://dx.doi.org/10.1016/S09608524(02)00013-5

Das N. y Mukherjee M. (2007). Cultivation of Pleurotus ostreatus on weed plants. Bioresource Technol. 98, 2723-2726. http://dx.doi.org/10.1016/j. biortech.2006.09.061

Díaz R. (2009). Efecto del pH inicial de desarrollo de Pleurotus ostreatus en fermentación sumergida sobre su actividad de lacasas. Tesis de Maestría. Centro de Investigación en Biotecnología Aplicada (CIBA-IPN). Instituto Politécnico Nacional. Tlaxcala, México, 108 pp

Delfín-Alcalá I. y Durán de Bazúa C. (2003). Biodegradación de residuos urbanos lignocelulósicos por Pleorotus. Rev. Int. Contam. Ambient. 19(1), 37-45.

Fanadzo M., Zireva D. T., Dube E. y Mashingaidze A. B. (2010). Evaluation of various substrates and supplements for biological efficiency of Pleurotus sajor-caju and Pleurotus ostreatus. Afr. J. Biotechnol. 9(19), 2756-2761. DOI: 10.5897/AJB2010.000-3100

Flores Ramírez G. (2012). Aprovechamiento del bagazo residual de Yucca spp. como sustrato para la producción de Pleurotus spp. Tesis de Maestría. Unidad Profesional Interdisciplinaria de Biotecnología. Instituto Politécnico Nacional. Ciudad de México, México, 111 pp.

Fracchia S., ArandaRickert A. y Terrizzano E. (2009). Cultivo de una cepa comercial de Pleurotus ostreatus en desechos de Simmondsia chinensis y Jatropha macrocarpa. Rev. Mex. Micol. 29, 37-42.

Gaitán-Hernández R., Salmones D., Pérez R. y Mata G. (2006). Manual práctico del cultivo de setas: aislamiento, siembra y producción. Instituto de Ecología, A.C. Xalapa, Ver., México. pp. 56.

García N., Bermúdez R. C., Gross P. y Hernández M.
(2006). Cultivo de cepas de Pleurotus sp. sobre pulpa de café. Rev. Mex. Micol. 23, 99-101.

Guzmán G., Mata G., Salmones D., Soto-Velazco C. y Guzmán-Dávalos L. (2008). El cultivo de los hongos comestibles, con especial atención a especies tropicales y subtropicales en esquilmos y residuos agro-industriales. Instituto Politécnico Nacional. D.F., México. pp. 245.

Heredia, C. (2010). Utilización de residuos de brócoli (Brassica olerácea itálica), coliflor (Brassica olerácea) y romanesco (Brassica olerácea botrytis) generados en la empresa PROVEFRUT S. A. para la producción de setas Pleurotus. Tesis de Ingeniería. Facultad de Ciencias e Ingeniería en Alimentos. Universidad Técnica de Ambato. Ambato, Ecuador, 97 pp.

Huerta G., Martínez-Carrera D., Sánchez J. E. y Leal-Lara H. (2009). Grupos de interesterilidad y productividad de cepas de Pleurotus de regiones tropicales y subtropicales de México. Rev. Mex. Micol. 30, 31-42.

Hurst C., Crawford R., Garland J., Lipson D., Mills A. y Stetzenbach L. (2007). Manual of environmental microbiology. AMS press. Washington D.C., United States of America. $1352 \mathrm{pp}$.

Ingale A. y Ramteke A. (2010). Studies on cultivation and biological efficiency of mushrooms grown on different agro-residues. Innov. Rom. Food. Biotechnol. 6, 25-28.

Jafarpour M., Zand A. J., Dehdashtizadeh B. y Eghbalsaied S. (2010). Evaluation of agricultural wastes and food supplements usage on growth characteristics of Pleurotus ostreatus. Afr. J. Agric. Res. 5, 3291-3296. DOI: 10.5897/AJAR10.623

Jwanny E. W., Rashad M. M. y Abdu H. M. (1995). Solidstate fermentation of agricultural wastes into food through Pleurotus cultivation. Appl. Biochem. Biotech. 50, 71-78. DOI: $10.1007 / \mathrm{BF} 02788041$

Kudryavtseva O. A., Dunaevsky Y. E., Kamzolkina O. V. y Belozersky M. A. (2008). Fungal Proteolytic Enzymes: Features of the Extracellular Proteases of Xylotrophic Basidiomycetes. J. Microbiol. 77, 643-653. DOI: $10.1134 / \mathrm{S} 0026261708060015$

Kumari D. y Achal V. (2008). Effect of different substrates on the production and non-enzymatic antioxidant activity of Pleurotus ostreatus (Oyster mushroom). Life Sci. J. 5, 73-76.

Kurt S. y Buyukalaca S. (2010). Yield performances and changes in enzyme activities of Pleurotus spp. (P. ostreatus and P. sajor-caju) cultivated on different agricultural wastes. Bioresource Technol. 101, 31643169. http://dx.doi.org/10.1016/j.biortech.2009.12.011

Lara M., Arias A. y Villaseñor L. (2002). Cultivation of Pleurotus ostreatus and P. pulmonarius on spent brewer's grain and tequila maguey bagasse. In: Proceed. IV International Conference on Mushroom Bi- 
ology and Mushroom Products, Cuernavaca, México. 323-330 pp.

Lechner B.E. y Albertó E. (2011). Search for new naturally occurring strains of Pleurotus to improve yields: Pleurotus albidus as a novel proposed species for mushroom production. Rev. Iberoam. Micol. 28, 148154. http://dx.doi.org/10.1016/j.riam.2010.12.001

Liang Z. C., Wu C. Y., Shieh Z. L. y Cheng S. L. (2009). Utilization of grass plants for cultivation of Pleurotus citrinopileatus. Int. Biodeter. Biodegr. 63, 509-514. http://dx.doi.org/10.1016/j.ibiod.2008.12.006

López-Rodríguez C., Hernández-Corredor R., SuárezFranco C. y Borrero M. (2008). Evaluación del crecimiento y producción de Pleurotus ostreatus sobre diferentes residuos agroindustriales del departamento de Cundinamarca. Univ. Sci. 13, 128-137.

Loss E., Royer A. R., Barreto-Rodrigues M. y Barana A. C. (2009). Use of maize wastewater for the cultivation of the Pleurotus spp. mushroom and optimization of its biological efficiency. J. Hazard Mater. 166, 15221525. http://dx.doi.org/10.1016/j.jhazmat.2008.11.014

Mamiro D. P. y Mamiro P. S. (2011). Yield and mushroom size of Pleurotus ostreatus grown on rice straw basal substrate mixed and supplemented with various crop residues. J. Anim. Plant Sci. 10, 1211-1218.

Mandeel Q. A., Al-Laith A. A. y Mohamed S. A. (2005). Cultivation of oyster mushrooms (Pleurotus spp.) on various lignocellulosic wastes. World J. Microbiol. Biotechnol. 21, 601-607. DOI: 10.1007/s11274-004-3494-4

Melo de Carvalho C. S., Sales-Campos C. y Nogueira de Andrade M. C. (2010). Mushrooms of the Pleurotus genus: a review of cultivation techniques. Interciencia. 35, 177-182.

Moda E. M., Horii J. y Fillet-Spoto M. H. (2005). Edible mushroom Pleurotus sajor-caju production on washed and supplemented sugarcane bagasse. Sci. Agric. 62, 127-132. http://dx.doi.org/10.1590/S010390162005000200006

Morales, P. (1987). Cultivo de Pleurotus ostreatus sobre la pulpa de cardamomo. Rev. Mex. Micol. 3, 71-73.

Mshandete A. M. y Cuff J. (2008). Cultivation of three types of indigenous wild edible mushrooms: Coprinus cinereus, Pleurotus flabellatus and Volvariella volvocea on composted sisal decortications residue in Tanzania. Afr. J. Biotechnol. 7, 4551-4562. DOI: 10.5897/AJB08.792

Naraian R., Sahu R. K., Kumar S., Garg S. K., Singh C. S. y Kanaujia R. S. (2009). Influence of different nitrogen rich supplements during cultivation of Pleurotus florida on corn cob substrate. Environmentalist. 29, 1-7. DOI: $10.1007 / \mathrm{s} 10669-008-9174-4$

Naraian R., Srivastava J. y Garg S. K. (2011). Influence of dairy spent wash (DSW) on different cultivation phases and yield response of two Pleurotus mushrooms. Ann Microbiol. 61, 853-862.

DOI: $10.1007 / \mathrm{s} 13213-011-0206-9$

Narh D. L., Obodai M., Baka D. y Dzomeku M. (2011). The efficacy of sorghum and millet grains in spawn production and carpophore formation of Pleurotus ostreatus (Jacq. Ex. Fr) Kummer. Int. Food Res. J. 18, 1092-1097.

Obodai M., Cleland-Okine J. y Vowotor K. A. (2003). Comparative study on the growth and yield of Pleurotus ostreatus mushroom on different lignocellulosic byproducts. J. Ind. Microbiol. Biotechnol. 30, 146-149. DOI: $10.1007 / \mathrm{s} 10295-002-0021-1$

Pérez J., Muñoz-Dorado J., De la-Rubia T. y Martínez J. (2002). Biodegradation and biological treatments of cellulose, hemicellulose and lignin: an overview. Int. Microbiol. 5, 53-63. DOI: 10.1007/s10123-002-0062-3

Pérez R. y Mata G. (2005). Cultivo y selección de cepas de Pleurotus ostreatus y P. pulmonarius en viruta de pino: obtención de nuevas cepas y evaluación de su producción. Rev. Mex. Micol. 20, 53-59.

Philippoussis A., Zervakis G. y Diamantopoulou P. (2001). Bioconversion of agricultural lignocellulosic wastes through the cultivation of the edible mushrooms $\mathrm{Ag}$ rocybe aegerita, Volvariella volvacea and Pleurotus spp. World J. Microbiol. Biotechnol. 17, 191-200. doi:10.1023/A:1016685530312

Ragunathana R. y Swaminathan K. (2003). Nutritional status of Pleurotus spp. grown on various agro-wastes. Food Chem. 80, 371-375. http://dx.doi.org/10.1016/ S0308-8146(02)00275-3

Rajak S., Mahapatra S. C. y Basu M. (2011). Yield, Fruit Body Diameter and Cropping Duration of Oyster Mushroom (Pleurotus sajor caju) Grown on Different Grasses and Paddy Straw as Substrates. Eur. J. Med. Plants. 1, 10-17.

Rashad M. M., Abdou H. M., Mahmoud A. E. y Nooman M. U. (2009). Nutritional Analysis and Enzyme Activities of Pleurotus Ostreatus Cultivated on Citrus Limonium and Carica Papaya Wastes. Aust. J. Basic Appl. Sci. 3, 3352-3360.

Ríos M. P., Hoyos J. L. y Mosquera S. A. (2010). Evaluación de los parámetros productivos de la semilla de Pleurotus ostreatus propagada en diferentes medios de cultivo. Rev. Fac. Cienc. Agropec. 8, 86-94.

Rivera Omen R.L., Martínez Mamián C. A. y Morales Velasco S. (2013). Evaluación de residuos agrícolas como sustrato para la producción de Pleurotus ostreatus. Revista Luna Azul 37, 89-100.

Rodríguez-Estrada A. E., Jimenez-Gasco M. M. y Royse D. J. (2009). Improvement of yield of Pleurotus eryngii var. eryngii by substrate supplementation and use of a casing overlay. Bioresource Technol. 100, 5270-5276. 
http://dx.doi.org/10.1016/j.biortech.2009.02.073

Rodríguez N. y Zuluaga J. (1994). Cultivo de Pleurotus pulmonarius (Fr.) Quél. en pulpa de café. Cenicafé 45, 85-92.

Romero O., Huerta M., Damián M. A., Macías A., Tapia A. M., Parraguirre J. F. C. y Juárez J. (2010). Evaluación de la capacidad productiva de Pleurotus ostreatus con el uso de hoja de plátano (Musa paradisiaca L., cv. Roatan) deshidratada, en relación con otros sustratos agrícolas. Agron. Costarricense 34: 53-63.

Royse D., Rhodes T., Ohga S. y Sanchez J. (2004). Yield, mushroom size and time to production of Pleurotus cornucopiae (oyster mushroom) grown on switch grass substrate spawned and supplemented at various rates. Bioresource Technol. 91: 85-91. http://dx.doi. org/10.1016/S0960-8524(03)00151-2

Salmones D., Mestizo-Valdéz L. y Gaitán-Hernández R. (2004). Entrecruzamiento y evaluación de la producción de las variedades de Pleurotus djamor (Fr.) Boedijn. Rev. Mex. Micol. 18, 21-26.

Salmones D., Mata G y Waliszewski K. N. (2005). Comparative culturing of Pleurotus spp. on coffee pulp and wheat straw: biomass production and substrate biodegradation. Bioresource Technol. 96, 537-544. http://dx.doi.org/10.1016/j.biortech.2004.06.019

Sánchez A., Esqueda M., Gaitán-Hernández R., Córdova A. y Coronado M. L. (2008). Uso potencial del rastrojo de tomate como sustrato para el cultivo de Pleurotus spp. Rev. Mex. Micol. 28, 17-24.

Sánchez C. (2009). Lignocellulosic residues: Biodegradation and bioconversion by fungi. Biotechnol. Adv. 27, 185-194. http://dx.doi.org/10.1016/j.biotechadv.2008.11.001

Sánchez, C. (2010). Cultivation of Pleurotus ostreatus and other edible mushrooms. Appl. Microbiol. Biotechnol. 85, 1321-1337. DOI: 10.1007/s00253-009-2343-7

Sánchez Velez C. A. (2013). Evaluación de la productivi- dad del hongo comestible Pleurotus ostreatus sobre un residuo agroindustrial del Departamento del Valle del Cauca y residuos de poda de la Universidad Autónoma de Occidente. Tesis de Pregrado. Facultad de Ciencias Básicas. Departamento de Ciencias Ambientales. Universidad Autónoma de Occidente. Santiago de Cali, Colombia, 94 pp.

Soto-Velazco C., Guzmán-Dávalos L. y Rodríguez O. (1989). Cultivo del hongo comestible Pleurotus ostreatus sobre bagazo de maguey tequilero fermentado y mezclado con paja de trigo. Rev. Mex. Micol. 5, 97-101.

Tisdale T. E., Miyasaka S. C. y Hemmes D. E. (2006). Cultivation of the oyster mushroom (Pleurotus ostreatus) on wood substrates in Hawaii. World J. Microbiol. Biotechnol. 22, 201-206. DOI: 10.1007/s11274-005-9020-5

Toledo M. (2008). Residuos de maíz y quinua como potenciales sustratos para el cultivo de hongos comestibles Pleurotus ostreatus. Tesis de Ingeniería. Facultad de Ciencias. Escuela de Ciencias Químicas. Escuela Superior Politécnica del Chimborazo. Chimborazo, Ecuador, $96 \mathrm{pp}$.

Varnero M. T., Quiroz M. S. y Álvarez C. H. (2010). Utilización de Residuos Forestales Lignocelulósicos para Producción del Hongo Ostra (Pleurotus ostreatus). Información Tecnológica. 21, 13-20. http://dx.doi. org/10.4067/S0718-07642010000200003

Velázquez-Cedeño M. A., Mata G. y Savoie J. M. (2002). Waste-reducing cultivation of Pleurotus ostreatus and Pleurotus pulmonarius on coffee pulp: changes in the production of some lignocellulolytic enzymes. World J. Microbiol. Biotechnol. 18, 201-207. DOI: 10.1023/A:1014999616381

Young R. y Akhtar M. (1998). Environmentally friendly technologies for the pulp and paper industry. John Wiley y Sons, Inc. New York, EUA. 577 pp. 